\title{
ROLE OF BACTERIAL INFECTION IN STROKE
}

\author{
Prashanth V. N1, Aravind G. $N^{2}$
}

${ }^{1}$ Assistant Professor, Department of General Medicine, Bangalore Medical College and Research Institute, Bangalore. ${ }^{2}$ Assistant Professor, Department of General Medicine, Bangalore Medical College and Research Institute, Bangalore.

ABSTRACT: BACKGROUND OF THE STUDY: Ischemic stroke is a common condition in clinical practice. There are various risk factors causing ischemic stroke. The risk factors can be modifiable or non-modifiable of them Diabetes, hypertension, smoking, dyslipidemia etc. are established risk factors. Acute and chronic infections are known to accelerate large vessel disease and can lead to atherothrombotic or embolic episodes resulting in ischemic strokes.

MATERIALS AND METHODS: The present study was conducted in the Department of Medicine, Victoria Hospital and Bowring and Lady Curzon Hospital, Bangalore Medical College, Bangalore. A total of 81 patients were studied. 41 were in study and 40 in control group. The study period was from October 2011 to September 2013. Statistical analysis for the association between infection and stroke was done using Chi square test/Fischer exact test and Odds ratio was calculated. A 'p' value $<0.05$ was taken as significant.

RESULTS: The prevalence of ischemic stroke in our study showed that $14.6 \%$ patients were below 40 years (Young stroke) and $85.4 \%$ patients were between $41-80$ years. Infection was seen in $22.2 \%$ of the patients with in 40 years and $77.8 \%$ were above 40 years. In the $40-60$ year age group, $55.5 \%$ of the patients had infection and $22.2 \%$ in the elderly (60-80yrs) age group. In the control group, $5 \%$ of the patients had infection and all were within $40 \mathrm{yrs}$.

CONCLUSION: Acute bacterial infections may contribute to be an independent risk factor the occurrence of ischemic stroke along with other established risk factors. Respiratory tract infection, especially with gram-positive cocci, is more commonly associated with large vessel stroke in anterior circulation.

KEYWORDS: BPSK Modulator, Convolutional Encoder, CORDIC, DS-CDMA/CI, FPGA.

HOW TO CITE THIS ARTICLE: Prashanth V. N, Aravind G. N. "Role of Bacterial Infection in Stroke". Journal of Evolution of Medical and Dental Sciences 2015; Vol. 4, Issue 89, November 05; Page: 15470-15478, DOI: 10.14260/jemds/2015/2212.

INTRODUCTION: Ischemic stroke is a common condition in clinical practice. There are various risk factors causing ischemic stroke. The risk factors can be modifiable or nonmodifiable. Of them Diabetes, hypertension, smoking, dyslipidemia etc., are established risk factors. Acute and chronic infections are known to accelerate large vessel disease and can lead to Athero-thrombotic or embolic episodes resulting in ischemic strokes.

Acute infections, especially bacterial infections pose a higher risk for ischemic cerebrovascular events like stroke. ${ }^{1}$ Various plausible mechanisms have been given to explain these events.

Respiratory tract infections, urinary tract infections, periodontal infections etc. are some of the commoner infections associated with acute ischemic strokes. The incidence of stroke can be anywhere between 24 to $43 \% .2,3$ when associated with infections. Most of the infections, as per various studies, are bacterial in origin, though viral infections are also incriminated to cause such vascular events.

Though studies have shown that ischemic strokes can occur independently in the background of an acute infection, the association is not definite or clear. Also, the exact mechanism is not clear.

Financial or Other, Competing Interest: None.

Submission 01-10-2015, Peer Review 03-10-2015,

Acceptance 21-10-2015, Published 05-11-2015.

Corresponding Author:

Dr. Prashanth V. N,

No. 9, Karmic Road, Shankarapuram,

Bangalore-560004

E-mail: drprashaanthvn2000@yahoo.com

DOI:10.14260/jemds/2015/2212.
Therefore further studies will be needed to establish acute infection as an independent risk factor for ischemic stroke.

The present study is done to find out the possibility of an acute infection as an independent risk factor for ischemic stroke.

\section{MATERIALS AND METHOD:}

SOURCE OF DATA: The present study was conducted in the Department of Medicine, Victoria Hospital and Bowring and Lady Curzon Hospital, Bangalore Medical College, Bangalore. Patients admitted to the hospital were taken for the study. The study was conducted after taking ethical committee clearance and after obtaining a valid written consent from both the patients and the control group.

\section{METHOD OF COLLECTION OF DATA:}

Definition of study and control groups:

Study Subjects: Forty one consecutive patients admitted to the hospital with a first episode of ischemic stroke within $48 \mathrm{hrs}$ from the onset, with or without clinical evidence of infection, satisfying the inclusion/exclusion criteria was taken for the study.

Control Subjects: Forty consecutive patients admitted to the hospital without an ischemic stroke, with or without clinical evidence of infection, satisfying the inclusion/exclusion criteria was taken as the control group.

Both the groups had patients with other risk factors for ischemic stroke like diabetes mellitus, hypertension, dyslipidemia, IHD, history of smoking and alcohol consumption. 
Inclusion Criteria: Symptoms like cough with expectoration, fever, sore throat, urinary symptoms like dysuria or hematuria, tooth pain, loose stools etc. and signs like, lymphadenopathy, pharyngitis, consolidation etc. were taken as evidence of infection clinically:

1. Patients with first episode of ischemic stroke within 48 hrs of onset, as confirmed by CT scan.

2. Adult of all ages and both the sexes were included.

3. Adults of all ages and both the sexes were included.

4. Patients with hypertension, diabetes, ischemic heart disease and dyslipidemia were included in both the groups.

5. Patients who smoke and consume alcohol were also included in the study.

6. Confirmation of infection at admission was by laboratory evidence like isolation of the organism by specific culture methods like blood culture, urine culture, sputum culture etc. were done.

\section{Exclusion Criteria:}

1. Recurrent ischemic stroke

2. Hemorrhagic stroke

3. Strokes following head injury

4. Stroke with evidence of meningitis or meningoencephalitis

5. Transient ischemic attacks

6. Stroke in patients with a definite clinical evidence of valvular heart disease

7. Patients who develop infection after the stroke.

8. HIV positive cases.

\section{Study Methods:}

1. The present study is a Case Control study.

2. The study period is from October-2011 to September 2013.

3. A total of 81 patients were taken for study. 41 patients were in the study group and the other 40 in the control group.

4. All these patients were evaluated thoroughly by clinical, radiological and laboratory methods. Risk factors common to both the groups except acute infection were "matched" to eliminate the possibility of confounding.

5. Only cases with laboratory evidence of infection were considered positive.

6. Patients with the evidence of infection were treated with appropriate systemic antibiotics based on the site and severity. Specific medications were given based on the need and supportive care extended to all the patients.

7. The outcome of the neurological status in the study group was done at the time of discharge.

All the patients were thoroughly assessed clinically at the time of admission and subsequently underwent relevant investigations. The following are the investigations done in the study;

\section{INVESTIGATIONS:}

- $\mathrm{Hb} \%, \mathrm{TC}, \mathrm{DC}, \mathrm{ESR}, \mathrm{CRP}, \mathrm{ASLO}$.

- Urea, creatinine and electrolytes.

- Urine routine and culture/sensitivity.

- Sputum for gram's stain, AFB, culture/sensitivity.

- Blood culture and sensitivity.
- Chest X-ray PA view.

- ECG.

- Mantoux test.

- Elisa for HIV.

- FBS and PPBS, Lipid profiles, liver function tests.

- CT Scan.

- Lumbar puncture for CSF.

- Stool examination (culture if needed).

- Miscellaneous.

All the patients received specific and supportive treatment and the laboratory data was analyzed for statistical relevance. The following is the detail of statistical methods used in the study.

Statistical Analysis: Statistical analysis for the association between infection and stroke was done using Chi square test/Fischer exact test and Odds ratio was calculated. A ' $p$ ' value $<0.05$ was taken as significant.

Statistical Methods: Chi-square and Fisher Exact test has been used to test the significant proportion of study characteristics between two groups. Student $t$ test (Two tailed, Independent) has been employed to test the significance of study parameters between the two groups of subjects.

Statistical software: The statistical software namely SPSS 11.0, Stata 8.0, Systat 11.0, Medcalc 9.0.1 and Effect Size calculator were used for the analysis of the data and Microsoft Word and Excel have been used to generate graphs, tables etc.

RESULTS: A total of 81 subjects were taken for the study after a formal written consent. Of them, 41 subjects were cases and 40 were controls. They were selected based on the inclusion and exclusion criteria.

The study was conducted over a period of 2 years (from Oct 2011-Sept. 2013) on 81 patients. Of them, 41 patients were in the study group and 40 were in the control group.

ANALYSIS OF THE STUDY: AGE DISTRIBUTION: The distribution of the patients in cases and control group is as follows:

There were 27 cases and 40 controls below the age 60 years, and 14 cases above the age of 60 years. That is, $65.8 \%$ of cases and all the controls were within 60 years. The remaining $34.2 \%$ cases were above the age of 60 years. In the cases, the youngest individual was $30 \mathrm{yrs}$. and the oldest being $80 \mathrm{yrs}$. with a Mean (SD) age of $55.95 \pm 12.85$ yrs.

However, in the control group, the youngest individual was 18 yrs. and oldest being 60 yrs. with a Mean age of $38.33 \pm 12.72$ yrs. That is, there is a difference of nearly 17 yrs. in the mean age between the cases and controls. Of the 41 cases studied, 31 were males and 10 were females; $\mathrm{M}$ : $\mathrm{F}$ ratio being 3:1. In the control group, there were 26 males and 14 females ( $n=40) ; \mathrm{M}: \mathrm{F}$ ratio 1.8:1 That is, in the present study, males were more than females in both the groups, but more amongst cases than controls. 


\section{SYMPTOM ANALYSIS:}

FEVER: 11 out of 41 cases (26.8\%) presented with fever at the time of admission while none of the controls had fever at admission $\left(\mathrm{p}<0.001^{*}\right)$. However, on examination 14 out of 41 cases (34.1\%) had fever. Only 6 out of 14 cases (42.8\%), proved to have infection by culture, had fever at admission. In the remaining 3 cases with infection but no fever, 2 of them had respiratory tract infection and one had urinary tract infection.

DYSURIA: 3 cases $(7.3 \%)$ had dysuria at the time of admission and 2 of them had fever (66.6\%). Only one patient had cough with dysuria (33.3\%) but no fever. ( $p=0.241)$. None of the controls had dysuria.

COUGH: 7 cases $(17 \%)$ and $2(5 \%)$ controls had cough with expectoration. 3 of the 7 cases were smokers (42.8\%). 5 of the 7 cases had fever (71.4\%) while there was no fever in the control group. 6 out of 7 cases (85.7\%) had positive sputum culture; the organisms isolated were beta hemolytic streptococci and Streptococcus pneumonia in 3 cases each. In the control group, 2 had cough with expectoration and 1 was a smoker (50\%). None had a positive sputum culture $(\mathrm{p}=0.155)$.

\section{RISK FACTOR ANALYSIS: SMOKING:}

Cases: 24 of the 41 cases were smokers (58.5\%). All were males $(100 \%)$. The minimum duration of smoking was $3 y$ rs and the maximum was 20yrs; Average pack years: 12 Average smoking index: 120. 14/24 were beedi smokers (58.3\%) and 10/24 were cigarette smokers (41.6\%). However, there was no difference in the outcome in the two groups.

Controls: 10 out of 40 controls were smokers (25\%); all were males (100\%). Minimum duration of smoking was $1 \mathrm{yr}$ and maximum was 15yrs. Average pack years: 8 .Average smoking index: 80 6/10 were beedi smokers, while 4/10 were smoking cigarettes.

It is seen that the patients in the study group smoked more than the control group and the comparative value is statistically significant $\left(\mathrm{p}<0.002^{*}\right)$

\section{ALCOHOLCONSUMPTION:}

Cases: 14 of $41(34.1 \%)$ were alcoholics; of them 13 were males and 1 was female. Minimum duration of alcohol consumption: 1yr. Maximum duration was 10yrs. Average duration was 5yrs. Average consumption was $100 \mathrm{~g} / \mathrm{d}$ for at least 3 days/wk.

Controls: 15 of 40 (37.5\%) controls were alcoholics; all were males. Minimum duration of alcohol consumption was 1yr. Maximum duration of consumption was 25yrs. Average consumption: $150 \mathrm{~g} / \mathrm{d}$ for at least 3 days/wk. 6/15(40\%) patients were admitted due to alcohol related problems. 2 for alcoholic intoxication, 3 for alcoholic liver disease and 1 for gastritis. On comparing the two groups, there was no statistically significant difference between them $(p=0.753)$.

HYPERTENSION: Cases: 25 out of 41 (60.9\%) patients had hypertension at admission (BP $>140 / 90 \mathrm{~mm} \mathrm{Hg}$ ). Of them, 21 (84\%) were known cases of hypertension.
$18 / 25$ were males $(72 \%)$ and $7 / 25$ were females (28\%) $19 / 25$ had hypertension of $<5 y$ rs duration (90.4\%), 2 had hypertension of $<6$ Months. 4/25 were freshly diagnosed cases of hypertension. (16\%) 16/21 were on regular medications for hypertension (76\%). 8/25 ha d type 2 DM along with hypertension (32\%). 10/25 had isolated systolic hypertension (40\%). 15/25 had both systolic and diastolic hypertension (60\%). 10/25 patients had Retinopathy (40\%); 8/10 had only hypertensive changes, while $2 / 10$ had both diabetic and hypertensive changes on fundus examination.

Controls: 10 out of 40 patients had hypertension (25\%). 6/10 were males and $4 / 10$ were females. $2 / 10$ had concomitant type 2 DM (20\%). 7/10 were on regular treatment for hypertension. All patients had both systolic and diastolic hypertension. 6/10 patients had retinopathy; $5 / 6$ had only hypertensive changes and $1 / 6$ had both diabetic and hypertensive change. It appears that the difference between the two groups with respect to hypertension is statistically significant $\left(\mathrm{p}<0.015^{*}\right)$. Therefore hypertension is a significant risk factor for ischemic stroke even in this study.

\section{DIABETES MELLITUS:}

Cases: 11 of 41 (26.8\%) patients were diabetics. 8/11 (72.7\%) were males and $3 / 11(27.3 \%)$ were females. 8 patients had both HTN and DM (72.7\%).2/8 patients had retinopathy changes on fundus examination (25\%). All of them had fasting hyperglycemia (100\%). 9 patients were known cases of Type 2 DM (90\%). One patient was detected to be diabetic after admission (10\%). 6/41 patients had Impaired fasting glucose (14.6\%). 6/41 patients had impaired glucose tolerance (14.6\%). $1 / 11$ diabetics expired subsequently (9\%). 2/6 patients with impaired fasting glucose died (33.3\%). The cause of death in all the three cases was aspiration pneumonia.

CONTROLS: 6 of the 40 controls were diabetics (type 2) (15\%). $5 / 6$ were males (83.3\%) and $1 / 6$ was female (16.7\%). 5 out of 6 patients had diabetes of <5yrs. $3 / 6$ patients had fasting hyperglycemia (50\%). 2 out of 6 patients had HTN also (33.3\%). Both these patients had features of retinopathy. 3/40 had impaired fasting glucose (7.5\%). 1/40 had impaired glucose tolerance $(2.5 \%)$. On comparing the two groups there was no statistically significant difference $(p=0.191)$.

DYSLIPIDEMIA: It appears that lipid profile has a significant influence on ischemic stroke in the present study. Especially LDL and triglycerides have highly significant $p$ values of 0.001

Cases: In the present study, 38/41 cases (92.6\%) had dyslipidemia; either one or all the parameters were elevated in them. Of the 9 cases with infection, 7 had a rise in more than one parameter (77.7\%). One each had a low HDL and an elevated VLDL levels respectively.

Controls: In the control group, 23/40 cases had dyslipidemia $(57.5 \%)$. Both the cases with infection had dyslipidemia. It is seen that LDL and Triglycerides were significantly elevated in the study group. LDL level was high in 27 cases $(65.8 \%)$ and 12 controls $(30 \%)\left(\mathrm{p}=0.001^{* *}\right)$. Similarly triglyceride was elevated in 28 cases $(68.3 \%)$ and 12 controls $(30 \%)\left(p=0.001^{* *}\right)$. 
Total cholesterol, VLDL and HDL was elevated in the study group than the control group but the difference was not statistically significant. On comparing the two groups, the $\mathrm{p}$ value was significant $\left(\mathrm{p}=0.001^{* *}\right)$ especially for $\mathrm{LDL}$ and triglycerides as shown in the table.

\section{Ischemic heart disease:}

Cases: 22/41 cases had various changes in ECG (53.6\%). Of them only 10 underwent 2D echo subsequently. 3 out of 10 patients had evidence of Ischemic heart disease in the form of $\mathrm{LV}$ dysfunction and regional wall motion abnormalities.

- $16 / 22$ cases were hypertensive (72.7\%).

- 16/22 had ST-T changes (72.7\%) not suggestive of Myocardial infarction.

- 9/16 cases had LV strain pattern on ECG.

- 5/22 had LVH (22.7\%); 2 patients had LV diastolic dysfunction.

- 1 patient had evidence of old inferior wall infarction.

- One patient had RBBB and another LAHB.

- $3 / 22$ had nonspecific changes.

Controls: 7/40 controls had ECG changes (17.5\%); all seven were hypertensive. $3 / 7$ had inferolateral ischemia (42.8\%). $1 / 7$ had transmural ischemia. $3 / 7$ had LVH (42.8\%).

It is seen that features of ischemic heart disease is present in both the groups, but the evidence of IHD is more in the study group.

ISCHEMIC STROKE: Of the 41 cases, 35 cases (85.3\%) had anterior circulation involvement and 6 cases $(14.6 \%)$ had posterior circulation stroke.

Anterior circulation: 30 out of 35 cases in this group had middle cerebral artery infarcts (85.7\%). 5 of the remaining cases had lacunar infarcts (14.3\%).

Posterior circulation: 2 of the 6 cases had (33.3\%) lacunar infarcts in the brainstem $4 / 6$ cases had (66.6\%) infarcts in the vertebrobasilar territory.

\section{CT SCAN REPORT:}

Anterior Circulation: 35 out of 41 cases (85.3\%) had infarcts in anterior circulation. All were in the middle cerebral artery territory. The most common sites were the Internal capsule, Frontotemporal and Temporoparietal areas:

- $13 / 35$ patients had significant cerebral edema (37.1\%).

- $24 / 35$ were on the left side (68.5\%) and 11 ere on right side $(31.5 \%)$.

- $\quad 5 / 35$ cases had lacunar infarcts (14.2\%) and 30/35 had large vessel infarcts (85.8\%).

\section{Posterior Circulation:}

- 6 out of 41 cases (14.7\%) had infarcts in posterior circulation.

- $2 / 6$ cases had lacunar infarcts; both of them in the PCA territory.

- 4/6 cases had large vessel infarcts; all of them in the vertebrobasilar territory.
- 2/4 were Medullary infarcts, one each of Midbrain and Pontine infarcts respectively.

\section{Cranial Nerve Involvement:}

- UMN type of facial palsy was the most common finding.

- $27 / 41$ cases had (UMN) facial involvement (65.5\%).

- $11 / 41$ cases had no cranial nerve involvement (26.8\%).

- $3 / 41$ had brainstem involvement (in the form of 9th, 10th, 11 th cranial nerve) (7\%).

- $1 / 41$ had 12 th nerve involvement (2\%).

\section{ACUTE PHASE REACTANTS:}

\section{C- REACTIVE PROTEIN: CRP}

35/41 cases had positive CRP levels (85.4\%) and

- $8 / 9$ (88.9\%) patients who had evidence of infection had positive CRP levels.

- 2/40 controls (5\%) had positive CRP levels and both of them $(100 \%)$ were subsequently found to have infection (urinary tract).

- In the present study, CRP levels were qualitatively assessed and the $p$ value was statistically significant $\left(\mathrm{p}<0.001^{* *}\right)$.

ASLO: $3 / 41$ cases (7.3\%) had significant ASLO titre ( $>300$ TU) $1 / 3$ cases had respiratory tract infection. None of the controls had positive ASLO titre. However, the $p$ value was not significant $(\mathrm{p}=0.241)$.

ESR: 31/41 cases had high ESR levels (75.6\%) and 8/31 cases (25.8\%) had infection. 10/40 controls had high ESR levels (25\%) and 2/4 controls had infection (50\%). 9/9 patients $(100 \%)$ who had infection, had high ESR levels at admission. 6/6 patients who died later also had high baseline ESR levels. The $p$ value is highly significant in the present study $\left(\mathrm{p}<0.001^{* *}\right)$ On comparing ESR with CRP, which was positive in 35 cases (85.4\%), ESR was high in two cases where CRP was negative.

Both these cases had infection. On comparing leucocyte count to ESR, 20/31 cases with high ESR had WBC count on the higher side of normal limit and 2 cases had leucocytosis.

Leucocytosis: The WBC count in the study group showed relative Neutrophilia (31/41 cases), but only one patient had leucocytosis $(>11,000$ cell/cumm). None of the controls had leucocytosis. However, the difference was not significant.

\section{INFECTION: Urine analysis:}

Cases: $25 / 41$ cases $(60.9 \%)$ had abnormalities in the form of either albuminuria or glycosuria. $16 / 41$ cases (39\%) had no abnormalities in urinalysis. 2/9 cases with infection had normal urinalysis $(22.2 \%) .3 / 9$ cases with infection had albuminuria only (1-2+) (33.3\%). 4/9 patients with infection had either glycosuria alone or with albuminuria (44.4\%). All 4 patients who had urinary tract infection had abnormalities on urinalysis, $3 / 4(75 \%)$ having plenty of pus cells and 1/4 (25\%) having only albuminuria.

Controls: $11 / 40(27.5 \%)$ controls had abnormal urine reports. $5 / 11$ had glycosuria $(45.4 \%)$ and $4 / 5$ also had albuminuria (80\%). 6/11 controls had only albuminuria (54.5\%). $2 / 11$ cases had urinary tract infection (18\%) and both were diabetics with 
glycosuria and albuniuria. On comparing the cases and controls, the $p$ value was statistically significant $\left(p=0.002^{* *}\right)$.

Sputum for gram stain: 6 cases (14.6\%) had positive gram stain reports. Of them, 3 patients had gram positive diplococci (Str. pneumonia) and other 3 had gram positive cocci in chains (streptococci). None of the controls had positive gram stain reports $\left(\mathrm{p}=0.026^{*}\right)$.

Culture Reports: 9 out of total 41 cases (21.9\%) and 2/40 (5\%) controls had evidence of infection on culture. Of the 9 cases, 6 had positive sputum culture $(66.6 \%)$ and 4 cases had positive urine culture $(44.4 \%)$. One patient had both sputum and urine culture being positive (11.1\%) and subsequently recovered following treatment.

Sputum Culture: 6/9 cases had positive sputum culture. $3 / 6(50 \%)$ had beta hemolytic Streptococci and the other 3 had Str. pneumoniae as the causative organism. None of the control group had any growth on sputum culture. The $p$ value was statistically significant in this aspect $\left(\mathrm{p}=0.026^{*}\right)$.

Urine Culture: 4 of the 9 cases had positive urine culture. $3 / 4$ cases were positive for E.coli $(75 \%)$ and $1 / 4$ had Proteus mirabilis infection. $2 / 2$ controls had urinary infection. One was positive for E.coli and the other was positive for Proteus mirabilis. On comparing the cases and controls the $\mathrm{p}$ value was not significant $(\mathrm{p}=0.675)$.

Blood Culture: None of the cases or the controls had any growth on blood culture.

Liver function tests: 9 out 41 cases $(21.9 \%)$ and 5 out of 40 controls (12.5\%) had elevation ( $>40$ IU). In AST level, 5 out 9 cases and 3 out of 5 controls were alcoholic. 2 out of 9 cases with AST elevation also had icterus. All of the controls had icterus along with AST elevation. 7 cases and 6 controls had elevated ALT levels. 5/7 cases and 3/6 controls were alcoholics. 2 out of cases had icterus while all the patients in the control group (6/6) had icterus. However, the difference between them was not statistically significant.

\section{Chest X ray: CXR:}

Cases: $12 / 41$ cases had $\mathrm{X}$ ray changes (29.2\%). Of them 3 had features of consolidation (25\%). 9/12 had features of COPD (75\%).

Controls: $5 / 40$ had $X$ ray changes $(12.5 \%)$ amongst the controls. All had cardiomegaly with $4 / 5$ of these patients being hypertensive.

OUTCOME: In the present study, it is seen that majority of the patients had partial recovery (65.9\%). 9.8\% patients made no recovery at all despite supportive therapy. $14.6 \%$ patients died and in 2/6 cases, death was due to septicemia $(33.3 \%)$ and in $4 / 6$ cases, death was due to aspiration pneumonia $(66.6 \%)$.

Infection and Outcome: Of the nine patients $(9 / 41 ; 21.9 \%)$ who had infection, 4 died (44.4\%) and 5/9(55.6\%) patients recovered. $4 / 5(80 \%)$ patients made a partial recovery with motor power of $3 / 5$ at discharge and $1 / 5(20 \%)$ made a good recovery following treatment with a power of $4+/ 5$ at discharge. 5/9 patients had fever of $<3$ days (55.5\%) and 4/9 cases had fever $>3$ days but $<7$ days. $1 / 9$ patient had no risk factors other than infection (11.1\%) and there was good recovery following treatment. 8/9 patients had one or more risk factors along with infection and majority of them had partial recovery. Thus, the present study shows that acute infections, most commonly bacterial in origin, of less than one-week duration may be associated with increased risk of ischemic stroke.

OUTCOME: MORTALITY: A total of 6 patients died in the study group while there was no mortality in the control group. 4 of them died due to Aspiration pneumonia (66.6\%) 2 patients died due to septicemia (33.3\%). 4 out of these 6 patients had infection (66.6\%) 5 out of 6 patients (cases, 83.3\%) who died subsequently had fever. Only 1 patient who died didn't have fever at admission. 2 patients who died from septicemia had fever and cough at onset. Also, both had respiratory tract infection. One patient was male and the other was female. One patient was a long-standing hypertensive and the other had no other risk factor.

The culture reports in both the cases showed Streptococcus pneumonia. Of the nine patients $(9 / 41 ; 21.9 \%)$ who had infection, 4 died (44.4\%) and 5/9(55.6\%) patients recovered. $4 / 5(80 \%)$ patients made a partial recovery with motor power of $3 / 5$ at discharge and $1 / 5(20 \%)$ made a good recovery with a power of $4+/ 5$ at discharge.

DISCUSSION: A case control study was done with 41 patients in the study group and 40 patients in the control group. The age distribution was different in the two groups; In the study group $30 \%$ of the subjects were within 50 years and $70 \%$ was in the 51-80 year age group. However the controls had all the patients below 60 years. That is, mean age in the control group was 17 years lesser than the study group.

The prevalence of ischemic stroke in our study showed that $14.6 \%$ patients were below 40 years (young stroke) and $85.4 \%$ patients were between $41-80$ years. Of them, $34.2 \%$ patients were above 60 years in the study group while 32.55 of the control group were less than 30 years.

The prevalence of infection was also different between the two groups. Infection was seen in $22.2 \%$ of the patients with in 40 years and $77.8 \%$ were above 40 years. In the $40-60$ year age group, $55.5 \%$ of the patients had infection and $22.2 \%$ in the elderly (60-80yrs) age group. In the control group, $5 \%$ of the patients had infection and all were within $40 \mathrm{yrs}$.

A study was done by Nagaraja et al. ${ }^{3}$ with the study population $<40 \mathrm{yrs}$, the risk of infection with respect to age is not comparable to the present study. In a study done by Grau et al, the study population was between 21-80 years; the study and control group was comparable as $53 \%$ were above $40 \mathrm{yrs}$ in the study and $48 \%$ in the control group. Patients in the 51-70yrs age group had evidence of infection in $57.8 \%$.

Bova et al..$^{2}$ showed that $32.8 \%$ of their study population was in the $65-75$ year age group and $23.2 \%$ in the 75 -85yrs age group.

These studies emphasize that infection can be a significant risk factor not only in young but also in the elderly. 
The sex distribution in our study showed clear male predominance in both the groups. In the study group, $75 \%$ were males and $65 \%$ of the controls were males. The male to female ratio in the study group was $3: 1$ and $1.8: 1$ in the control group. In patients with infection $33.3 \%$ was seen in females and $66.6 \%$ in the males. In the control group, all patients with infection were males.

Paginini et al. had a comparable male to female population in the cases and control group. However, infection was common amongst the males $(63.1 \%$ Vs $36.9 \%)$. Grau et al also showed similar results with respect to sex distribution and infection (18.1\% Vs 6.0\%). Nagaraja et al. $^{3}$ had a male to female ratio of $1.2: 1$ in the study group and 1.1:1 in the controls.

Fever was seen in $34.1 \%$ of the study group and none in the control group. All the patients had fever of less than 7 days duration with $63 \%$ of them having fever for less than 3 days. In the patients $42.8 \%$ with fever had infection with ischemic stroke and $33.3 \%$ of the patients with infection did not have fever at admission. Two out of three patients with infection, who did not have fever, had urinary tract infection and one had respiratory tract infection. In patients with fever, $84.6 \%$ had anterior circulation stroke and $15.4 \%$ had posterior circulation. Of them, $77 \%$ had large vessel involvement and $23 \%$ had lacunar infarcts.

Grau et al. ${ }^{1}$ showed that $63 \%$ of their patients had fever and majority of them had fever of $<7$ days duration; in these patients $42.1 \%$ had infection. Nagaraja et al showed that $23.3 \%$ of the patients had fever and $28.5 \%$ of those patients had evidence of infection.

Dysuria was present in $7.3 \%$ patients in the study group. Of them, $66.6 \%$ had urinary tract infection. While none of the control patients had dysuria, urinary tract infection was present in $5 \%$ of them and all of them were diabetics. Cough was present in $17.1 \%$ of the cases out of which $42.8 \%$ were smokers. In the study group $42 \%$ had respiratory tract infection. $5 \%$ of the controls had cough but none had evidence of infection 4,5

Smoking is one of the commonly associated risk factors for ischemic events like stroke. There were 58.5\% smokers in the study group all were males. In smokers $16 \%$ had respiratory infection. There were $25 \%$ smokers in the control group but none had any infection.

Nagaraja et al. ${ }^{3}$ showed that $26.7 \%$ of their cases were smokers and $31.7 \%$ of them had respiratory infection. However, the duration of smoking was not proportionate to either stroke or infection.

Grau et al. ${ }^{1}$ in their study had $24.3 \%$ of smokers in the study group with $28 \%$ of them developing respiratory tract infection subsequently. The control group had 19.7\% smokers with only $11 \%$ having infection. Respiratory followed by dental infections was common amongst smokers in this study. Paginini et al. ${ }^{5}$ showed $27 \%$ of the cases were smokers and $28 \%$ of them had respiratory infection and $16.1 \%$ had dental infection.

Alcohol consumption is associated with atherosclerotic complications. $34 \%$ of the cases and $37.5 \%$ controls were alcoholics.

In the study group, $22.2 \%$ had infection, all being respiratory tract infections. In the control group, both the patients with infection were alcoholics and they had urinary Journal of Evolution of Medical and Dental Sciences/ eISSN- 2278-4802 infection unlike the study group. The duration and quantity of alcohol consumption was not related to either stroke or infection in this study.

Grau et al. ${ }^{1}$ showed that $24 \%$ of their study group were alcoholics and $21.2 \%$ of them had infection. These patients had both respiratory and urinary tact infection in equal proportion. Nagaraja et al. ${ }^{3}$ also showed that $20 \%$ of the study population was alcoholics and all were males in their study.

Hypertension was present in $60.9 \%$ of the cases. Of them $84 \%$ were known cases of hypertension and $67 \%$ were on regular treatment; $17 \%$ were on irregular treatment. In the study group $90 \%$ had uncontrolled hypertension at admission, and $10 \%$ had normal blood pressure despite hypertension. Accelerated hypertension was present in $8 \%$ and $16 \%$ of the patients were found to have infection in the study group.

Anterior circulation stroke was present in $84 \%$ of the patients and $16 \%$ had posterior circulation stroke. Lacunar infarcts constituted $20 \%$ of the strokes and $80 \%$ had large vessel involvement. In the control group $25 \%$ had hypertension but none had infection.

Grau et al. ${ }^{1}$ showed $58.8 \%$ patients had hypertension and $65.8 \%$ of them had infection. The study did not mention regarding the type of stroke in hypertensive patients. Paginini et al. showed that $67 \%$ of their study subjects had hypertension and $52 \%$ of them had infection. Uncontrolled hypertension was seen in $48 \%$ patients.

Diabetes mellitus is an established risk for stroke. $26.8 \%$ of the cases and 155 of the controls had Type II diabetes. All the patients in both the groups were on oral hypoglycemic agents; impaired fasting glucose was seen in $9 \%$. Urinary tract infection in diabetics was seen in $11 \%$ and $36.6 \%$ patients had poorly controlled diabetes. Anterior circulation stroke was seen in $81 \%$ and $19 \%$ had posterior circulation involvement. Diabetes was poorly controlled in the control group, with all the patients having infection being diabetics.

Grau et al. ${ }^{1}$ in their study showed $37 \%$ of the cases had diabetes. $50 \%$ of the cases had uncontrolled diabetes. Infection was seen in $26.3 \%$ with respiratory tract being the commonest site of infection. Paginini et al showed $41 \%$ patients were diabetics and $44 \%$ of these cases had skin and dental infection.

Dyslipidemia was seen in $92.6 \%$ of the cases. LDL (65\%) and triglycerides (68.3\%) were elevated more often than other parameters. Of them $39 \%$ were smokers, $18 \%$ were diabetics and $55.2 \%$ were hypertensive; High cholesterol levels was seen in $36.6 \%, 19.5 \%$ had elevated VLDL levels and $48.8 \%$ of the patients had low HDL levels with male predominance. Infection was seen in $18.4 \%, 80 \%$ of which was in the respiratory tract. The difference between the study and control group was statistically significant with respect to LDL and triglycerides.

In a study by Grau et al. ${ }^{1} 30 \%$ patients had dyslipidemia with $70 \%$ having elevated LDL and triglyceride levels. Infection was present in $21.2 \%$ and $26.3 \%$ of these cases were diabetics. Bova et al. ${ }^{2}$ showed $13.2 \%$ of the cases had infection with dyslipidemia. In this study, they found total cholesterol and triglycerides to be elevated more often than LDL. Diabetes was present in $34 \%$ and $60.4 \%$ had hypertension.

Ischemic heart disease, one of the most common risk factors for various ischemic events including stroke was seen in $53.6 \%$ of the patients. ${ }^{6,7}$ Though none of our patients complained of angina or other symptoms suggestive of ischemia, most of these patients were diagnosed to have IHD based on 
either ECG or Echocardiography. Of these patients who underwent Echo, 30\% were found to LV dysfunction, regional wall motion abnormalities and other features suggestive of ischemia. Hypertension was present in $72.3 \%$ and $34 \%$ were diabetics. Infection was present in $9 \%$ of these patients.

Grau et al. ${ }^{1}$ showed that $28.4 \%$ of their study population had IHD and $22.2 \%$ had evidence of infection. Diabetes there in $15 \%$ of them, $22 \%$ had hypertension and $21.2 \%$ had dyslipidemia. The severity or site of stroke was not influenced by the presence of IHD in this study. Bova et al. ${ }^{2}$ showed that $34.1 \%$ of the cases had IHD with infection and $13.1 \%$ of them had associated dyslipidemia.

The predilection to either anterior or posterior circulation stroke was also studied. Accordingly, $85.3 \%$ had anterior circulation strokes, with all of them having infarcts in the Middle cerebral artery territory.

Infarct on the left side was seen in $68.5 \%$ and the remaining on the right side. Significant cerebral edema as evidenced by CT scan was seen in $37.1 \%$. Large vessel infarct was seen in $85.8 \%$ and $14.2 \%$ had lacunar infarcts. $20 \%$ of the patients with anterior circulation stroke had evidence of infection; of them $14 \%$ had urinary infection and $86 \%$ had respiratory tract infection.

Posterior circulation infarct was seen in $14.2 \%$ of the patients, and $33 \%$ was in the PCA territory. The remaining had brain stem infarcts. Large infarcts constituted $66.6 \%$ and $33.3 \%$ had lacunar infarcts. One third of the patients with posterior infarcts had infection, in respiratory as well as urinary tract.

Paginini et al. showed that $65 \%$ of their cases had anterior circulation with $44 \%$ lacunar infarcts and the remaining as large vessel infarcts. The most common infection in this study was dental infection along with respiratory tract.

Facial nerve was the most common cranial nerve to be involved in this study. $65 \%$ of the MCA infarcts had UMN type of facial palsy. UMN type of facial palsy in infection associated strokes was seen in $71.4 \%$.

Lower cranial nerves were involved in two thirds of the posterior circulation strokes, with $50 \%$ of them involving the $9^{\text {th }}, 10^{\text {th }}, 11^{\text {th }}$ cranial nerves. One patient had right-sided Claude's syndrome ( $3^{\text {rd }}$ nerve) and another pontine infarction.

Most of the infection related strokes with cranial nerve involvement had respiratory tract infection. Paginini et al. showed no particular significance to cranial nerve involvement with respect to infection related stroke in their study. ${ }^{8}$

C-reactive protein is an acute phase reactant that can be elevated in both infective as well as inflammatory conditions. Of the patients with stroke, $85.4 \%$ had positive CRP values in this study. CRP was assessed qualitatively in this study. $88.9 \%$ of infection related stroke had positive CRP values and $85 \%$ of the infections were in the respiratory tract; the remaining was in the urinary tract. CRP was positive in $55.5 \%$ of patients with posterior infarcts and $65 \%$ of the anterior (MCA) infarcts. Of the infection related. strokes $11.1 \%$ were negative for CRP. The severity of the stroke was not influenced by CRP levels in this study.
In patients with positive CRP levels $75.6 \%$ also had high ESR levels and only $9 \%$ patients had leucocytosis. Nagaraja et al. ${ }^{3}$ showed $38.3 \%$ of the cases had positive CRP values and $43.4 \%$ of these patients had infection.

ASLO levels were high in $7.3 \%$ of the patients and one third of these patients had respiratory tract infection. The isolate was beta hemolytic streptococci in these patients. All of them were positive for CRP had ESR was elevated in two thirds of these patients.

ESR was high in $75.6 \%$ of the patients and $35.8 \%$ of them had evidence of infection. Of the 9 cases with infection, all of them had high ESR levels. Also, all the patients who died subsequently had high ESR levels. In cases with high ESR levels $85.4 \%$ also had positive CRP levels. Only 9\% had associated leucocytosis.

Nagaraja et al. ${ }^{3}$ showed that $30 \%$ of their patients had high ESR and CRP levels and 65\% of them had high ESR levels. Grau et al. ${ }^{1}$ showed that $71 \%$ of the patients with stroke had high ESR levels and $55 \%$ of them had infection.

Leucocytosis was seen in only $25 \%$ of the patients and $95 \%$ of them had neutrophilia. $40 \%$ of the patients had infection and one patient had leucopenia despite infection. Of the patients who died $80 \%$ had polymorphonuclear leucocytosis. All patients with leucocytosis had positive CRP levels and 88\% had high ESR levels.

Nagaraja et al. ${ }^{1}$ showed that $20 \%$ of their patients had leucocytosis and $25 \%$ of these patients had infection. However, there was no relation to mortality in their study.

Urine examination showed that $60 \%$ of the study subjects had abnormalities in the form of albuminuria, glycosuria and pyuria. Fasting glycosuria was present in $20.9 \%$ and three fourths of them were diabetics. Albuminuria, was present in $43 \%$ (Not of nephrotic range) and $75 \%$ of these patients were found to have urinary tract infection. $85 \%$ of the patients with albuminuria had hypertension and majority of them were uncontrolled. Grau et al. ${ }^{1}$ showed no particular significance with respect to urine analysis in their study.

Acute infection, which is presently being considered as one of the possible risk factors for ischemic stroke was seen in $21.9 \%$ of the patients in the study group and $5 \%$ of the control group. In the study group $66 \%$ had respiratory tract infection and $34 \%$ had urinary tract infection.

Sputum examination showed that $66.6 \%$ of the patients with respiratory infection had gram-positive cocci; on culture, they yielded beta hemolytic Streptococci in 50\% of them and Streptococcus pneumonia in the remaining cases.

Urine culture showed $75 \%$ of the isolate was E.coli and remaining was Proteus mirabilis. In the control group, only 5\% of the patients had infection. All of them were in the urinary tract. E, coli and Proteus was isolated in those cases. Blood culture was negative in both the groups. None of the cases in either group had evidence of viral infection in the study9,10.

Anterior circulation stroke was seen in $77.7 \%$ of the cases with infection and the remaining in the vertebrobasilar territory. In the MCA territory, $85 \%$ patients had large infarcts and $15 \%$ had lacunar infarcts. However, in the posterior circulation, 25\% had lacunar infarct and $75 \%$ had large infarcts. Large vessel involvement was observed in $80 \%$ of the patients with respiratory tract infection and $20 \%$ had lacunar infarcts. 
In patients with urinary infection, $50 \%$ had large vessel stroke and $50 \%$ had lacunar infarcts.

Grau et al. ${ }^{1}$ showed that $60 \%$ of the subjects had bacterial infection, followed by $28.9 \%$ of viral infection and the remaining were fungal infections. Most common site of infection was respiratory tract followed by urinary tract. The most common organisms were Str. Pneumonia, Staph. aureus and Streptococcus.

Bova et al..$^{2}$ showed that of the $24.2 \%$ of the cases with infection, $40.9 \%$ cases were RTI, $31.8 \%$ were UTI. The most common isolate was Str. pneumonia.

Paginini et al. showed that $18.7 \%$ patients with stroke had infection. Of them, RTI, UTI, dental and skin infections were common. Large vessel stroke was seen in $44 \%$ and $36 \%$ had lacunar infarcts. Anterior circulation stroke constituted $65 \%$ and $35 \%$ of the strokes were in posterior circulation.

Nagaraja et al. $^{3}$ showed $43.3 \%$ of the cases had infection; most common site being the respiratory tract and the most common isolate being beta hemolytic Streptococci. More than $95 \%$ of the cases had large vessel stroke and most of them had anterior circulation involvement. Also, majority of the infections were less than one week in this study, which is comparable to the present study. The result of our study is comparable to other studies with this respect.9,10

Serum transaminases (AST/ALT) are markers of not only liver pathology but also that of inflammation. $21.9 \%$ of the cases and $12.5 \%$ of the controls had elevated transaminases. Of these patients $55.5 \%$ were alcoholics. In patients with infection $89 \%$ had normal AST/ALT levels. Nagaraja et al. ${ }^{3}$ showed that $21.6 \%$ of their patients had elevated AST/ALT levels but their relation to infection was not specific.

Chest radiography showed that $29.2 \%$ of the patients had X-ray abnormalities. Feature of consolidation was seen in $25 \%$ and $72 \%$ patients had COPD changes. Of the patients with respiratory infection, $50 \%$ had normal X-ray.

The recovery in the study subjects was variable. $48.8 \%$ patients had partial improvement in their motor power and majority of them took nearly 5 days for recovery. $17.1 \%$ had poor recovery, $9.8 \%$ had good recovery and $14.1 \%$ patients died. $44.4 \%$ of the patients with infection died. Of them, $50 \%$ died of aspiration pneumonia and the other half due to septicemia. Of the patients who died $75 \%$ had respiratory tract infection and $25 \%$ had urinary infection. In the patients $66.6 \%$ had anterior circulation stroke and $33.3 \%$ had posterior circulation stroke. Most of the deaths $\left(2 / 3^{\text {rd }}\right)$ occurred on the $5^{\text {th }}$ day of admission while one patient died on the $10^{\text {th }}$ day.
Grau et al. ${ }^{1}$ showed that the mortality was more common in patients with respiratory infection and those with large vessel involvement. However, the severity of the stroke was not related to infection as per this study.

\section{CONCLUSION:}

- Ischemic stroke is precipitated by several risk factors.

- Acute bacterial infections may contribute to the occurrence of ischemic stroke along with other established risk factors.

- Respiratory tract infection, especially with gram-positive cocci, is more commonly associated with large vessel stroke in anterior circulation.

- Further studies are needed to understand the pathophysiology.

\section{BIBLIOGRAPHY:}

1. Grau AJ, Buggle F, Steichen-Wiehn C, Heindl S, Banerjee T, Maiwald $\mathrm{M}$ et.al. Recent infection as risk factor for cerebrovascular ischemia. Stroke 1995; 26: 373-379.

2. Bova IY, Bornstein NM, Korczyn AD. Acute infection as a risk factor for ischemic stroke. Stroke 1996;27:2204-2206.

3. Nagaraja D, Christopher R, Tripathi M, Kumar MV, Valli ER, Patil SA. Preceding infection as a risk factor of stroke in the young. J Assoc Physicians India. 1999 Jul; 47 (7): 673-5.

4. Quest DO. Stroke a selective history. Neurosurgery 1990; 27: 440-445.

5. Paganini-Hill, Lozano E, Fischberg G, Perez Barreto M, et. al. Infection and the risk of spontaneous cervical artery dissection: A case-control study. Stroke 2003 July 1; 34 (7): e79-81.

6. Ginsberg MD, Bogousslavsky J, eds. Cerebrovascular Disease: Pathophysiology, Diagnosis, and Management. Vol. II. Malden, Mass: Blackwell Science; 1998: 1639-1640.

7. Allan Ropper, Robert Brown. Adam and Victor's Principles of Neurology: 8th edition, McGraw Hill Publications 2005; 663664.

8. Guillon B, Berthet K, Benslamia L, Bertrand M, Bousser M-G, Tzourio C. Inflammation and infections as risk factors for ischemic stroke. Stroke 2003 October 1; 34 (10): 2518-2532.

9. Lindsberg PJ and Grau AJ. Platelet-leukocyte interaction and platelet activation in acute stroke with and without preceding infection. Arterioscler Thromb Vasc Biol 2005 July 1; 25(7): 1519-1523.

10. Grau AJ, Buggle F, Lichy C, Brandt T, Becher H, Rudi J. Helicobacter pylori infection as an independent risk factor for cerebral ischemia of atherothrombotic origin. J Neurol Sci. 2001; 186: 1-5. 


\begin{tabular}{|c|c|c|}
\hline $\begin{array}{c}\text { Risk } \\
\text { Factors }\end{array}$ & $\begin{array}{c}\text { Lacunar } \\
\text { Infarcts (n=5) }\end{array}$ & $\begin{array}{c}\text { Large Vessel } \\
\text { Infarcts (n=30) }\end{array}$ \\
\hline Smoking & 5 & 19 \\
\hline Alcohol & 2 & 12 \\
\hline Hypertension & 2 & 19 \\
\hline Diabetes mellitus & 2 & 9 \\
\hline Infection & 1 & 6 \\
\hline Site of infection & Urinary tract & 5 respiratory tract (RTI) \\
1 urinary tract and RTI
\end{tabular}

\begin{tabular}{|c|c|c|}
\hline Culture & $\begin{array}{c}\text { Cases (n=41) } \\
\text { (Infection; 9 +ve) }\end{array}$ & $\begin{array}{c}\text { Controls (n=40) } \\
\text { (Infection; 2 +ve) }\end{array}$ \\
\hline 1. Sputum & $6(14.6 \%)$ & 0 \\
\hline Isolates & $\begin{array}{c}\text { Beta hemolytic streptococci } \\
3 / 6(50 \%)\end{array}$ & - \\
\hline & $\begin{array}{c}\text { Streptococcus pneumonia } \\
3 / 6(50 \%)\end{array}$ & $2(5 \%)$ \\
\hline 2.Urine & $4(9.8 \%)$ & E. coli $(1 / 2 ; 50 \%)$ \\
\hline Isolates & E. coli $(3 / 4 ; 75 \%)$ & - \\
\hline & Proteus mirabilis $(1 / 4 ; 25 \%)$ & Proteus mirabilis $(1 / 2 ; 50 \%)$ \\
\hline Both urine and sputum +ve & $1(1 / 9 ; 11.1 \%)$ & - \\
\hline \multicolumn{2}{|c|}{ Table 2: Comparison of culture reports between cases and controls } \\
\hline
\end{tabular}

